\title{
Polarization study of massive binaries with the ARIES-1.04 m telescope
}

\author{
Bharti Arora $^{1 *}$, Jeewan Chandra Pandey ${ }^{1}$, Arti Joshi ${ }^{1}$, Michaël De Becker $^{2}$ \\ ${ }^{1}$ Aryabhatta Research Institute of Observational Sciences (ARIES), Nainital-263 002, India \\ ${ }^{2}$ Space Sciences, Technologies and Astrophysics Research (STAR) Institute, \\ University of Liège, Quartier Agora, 19c, Allée du 6 Août, B5c, B-4000 Sart Tilman, Belgium
}

\begin{abstract}
We present preliminary results obtained from optical (B, V, R, and I) linear polarimetric observations of the $\mathrm{O}+\mathrm{O}$ massive binary DH Cep. The observations were made on six nights in 2017 with the Aries IMaging POLarimeter (AIMPOL) mounted at the back of the 1.04-m Sampurnanand Telescope of ARIES. The average intrinsic linear polarization in each of the $\mathrm{B}, \mathrm{V}, \mathrm{R}$, and I photometric bands is found to be less than $1 \%$. The degree of polarization as well as the polarization angle appear to be orbital phase dependent. The significant polarization variability noticed towards shorter wavelengths (i.e. B and V bands) is indicative of the asymmetric circumstellar envelope.
\end{abstract}

Keywords: Stars: early-type - Optical wavelength: stars - massive binaries: individual - DH Cep: techniquelinear polarization

\section{Introduction}

Massive stars (O-type and Wolf-Rayet stars) are hot and luminous objects which are characterized by their huge stellar winds that lead to high mass loss rates $\left(10^{-7}\right.$ to $10^{-5} \mathrm{M}_{\odot}$ per year). These stellar winds are dense and strongly ionized, thus abundant in free electrons and ions. This fact opens the door to a promising way of systematically probing the wind structure of these stars, namely via the polarization of starlight. In particular, free electrons can lead to linear polarization of light through single Thomson scattering in an ionized and optically thin plasma envelope co-rotating with the star (St.-Louis et al. 1988).

Most stars have a spherically symmetric circumstellar envelope, and therefore no intrinsic linear polarization is observed. However, for massive stars, which are known to have anisotropic winds as a result of the inherent instability of their line-driven mass loss, this is not the case. Further, this effect is even more significant in the case of short-period massive binaries, where the close companion acts like a probe and modulates the linear polarization as it orbits around the primary star and illuminates different regions of the electron-rich wind, e.g., CQ Cephei (WN6+O9, period-1.64 d, Drissen et al. 1986) and V444 Cyg (WN5+O6, period-4.21 d, St.-Louis et al. 1993). Some of the massive binaries have also been identified as dust producers despite their strong radiation fields. Their dust

*e-mail: bharti@aries.res.in 
formation is associated with the presence of strong colliding winds. Hence, these are expected to be highly polarized due to the free-free scattering. Polarimetry is, therefore, an important observational technique to study the dust formation and stellar winds of massive binaries.

We have initiated a polarimetric observation campaign of a sample of 12 massive binaries consisting of $\mathrm{WR}+\mathrm{O}$ as well as $\mathrm{O}+\mathrm{O}$ systems. Here, we will present preliminary results obtained for the $\mathrm{O}+\mathrm{O}$ binary $\mathrm{DH}$ Cep.

DH Cep (HD 215835) is a double-lined spectroscopic binary system. It is a member of the cluster NGC 7380 (age $\approx 4 \mathrm{Myr}$ ) and has an orbital period of $2.11 \mathrm{~d}$ (Lata et al. 2016). This binary star is also an X-ray source with $\log \left(L_{X} / L_{b o l}\right)$ of -6.7 in $0.3-7.5 \mathrm{keV}$ energy band, perhaps attributed to colliding winds. The presence of the cool $(<1 \mathrm{keV})$ and hot $(>1.89 \mathrm{keV})$ temperature components in $\mathrm{X}$-ray spectra of DH Cep could possibly be associated with the instabilities in radiation-driven wind shocks (Bhatt et al. 2010). The main orbital parameters of DH Cep are listed in Table 1.

Table 1: Basic parameters of the DH Cep system.

\begin{tabular}{cccc}
\hline \hline & \multicolumn{2}{c}{ DH Cep } & \\
Parameter & Primary & Secondary & Reference \\
\hline Distance (kpc) & \multicolumn{2}{c}{$3.2 \pm 0.4$} & 1 \\
$V$ (mag.) & \multicolumn{2}{c}{8.61} & 2 \\
Period (d) & \multicolumn{2}{c}{2.11} & 2 \\
eccentricity & \multicolumn{2}{c}{0} & 2 \\
$q\left(M_{1} / M_{2}\right)$ & \multicolumn{2}{c}{$1.15 \pm 0.02$} & 2 \\
$T_{o}(\mathrm{HJD})$ & $2456525.564 \pm 0.006$ & 2 \\
Spectral type & O5.5 V-III & O6 V-III & 2 \\
$\gamma\left(\mathrm{km} \mathrm{s}^{-1}\right)$ & $-46.43 \pm 2.47$ & $-51.95 \pm 2.65$ & 2 \\
$K\left(\mathrm{~km} \mathrm{~s}^{-1}\right)$ & $234.81 \pm 3.97$ & $269.70 \pm 4.56$ & 2 \\
$a \sin i\left(\mathrm{R}_{\odot}\right)$ & $9.79 \pm 0.17$ & $11.24 \pm 0.19$ & 2 \\
$\mathrm{M} \sin ^{3} i\left(\mathrm{M}_{\odot}\right)$ & $15.01 \pm 0.66$ & $13.07 \pm 0.57$ & 2 \\
\hline
\end{tabular}

Notes: $T_{o}$ refers to the time of the conjunction (primary in front). The parameters $\gamma, K, a \sin i$, and $\mathrm{M}$ $\sin ^{3} i$ denote the apparent systemic velocity, the semi-amplitude of the radial velocity curve, the projected distance from the center of the star to the center of mass of the binary system, and the minimum masses of the binary components, respectively.

References: (1) Lindegren et al. (2018), (2) Martins et al. (2017)

\section{Observations and data reduction}

The optical polarimetric observations of massive binaries were acquired using AIMPOL mounted at the Cassegrain focus of the 1.04-m Sampurnanad telescope of ARIES. The telescope is an RC reflector with a focal ratio of $\mathrm{f} / 13$. AIMPOL is coupled with a TK $1 \mathrm{~K} \times 1 \mathrm{~K} C \mathrm{CD}$ camera which is cooled by liquid- $\mathrm{N}_{2}$. AIMPOL consists of an achromatic half-wave plate (HWP) modulator and a Wollaston prism beam-splitter. Hence, for each object within the field-of-view, two images (ordinary and extra-ordinary) are formed. After alignment of the optical axis of AIMPOL with the North-South (NS) direction of the telescope, observations were done at four positions of $\mathrm{HWP}\left(0^{\circ}, 22.5^{\circ}, 45^{\circ}\right.$, and $\left.67.5^{\circ}\right)$.

The standard aperture photometry technique was used to estimate fluxes of the ordinary and extraordinary beams of the target at each position of HWP with the IRAF package. Two normalized 
Stokes parameters, Q and U, were calculated corresponding to the four positions of the HWP (see Rautela et al. 2004).

DH Cep was observed in the B, V, R, and I photometric bands having $\lambda_{\text {eff }}=0.44,0.55,0.66$, and $0.80 \mu \mathrm{m}$, respectively, on the nights of 15, 16 October, and 12, 20, 21 November, as well as 14 December, 2017. Polarized and un-polarized standard stars from Schmidt et al. (1992) were also observed to perform the zero point calibration of the estimated polarization angles of the target and the correction for instrumental polarization, respectively.

\section{Preliminary Results}

The variations of the degree of polarization and the polarization angle of DH Cep with orbital phase are shown in Fig. 1. The phase-dependent modulations are clearly visible in these light curves, specifically, in the $\mathrm{B}$ and $\mathrm{V}$ bands. The amplitude of the degree of polarization modulations is $\sim 0.7 \%$ in these bands. The variation of the polarization light curve as well as the polarization angle with wavelength point towards the intrinsically polarized nature of DH Cep. In order to estimate the interstellar linear polarization $(p)$ towards DH Cep, we plotted $p$ versus distance in Fig. 2. The $p$ values of the stars lying within a 2 deg field of DH Cep were taken from Heiles (2000) while their distances were retrieved from the Gaia DR2 archive (Lindegren et al. 2018). It is anticipated that $p$ increases linearly with the distance because of the presence of the interstellar medium. At the location of DH Cep (distance $=3.24 \mathrm{kpc}$ ), the $p$ value is $\sim 2 \%$. Therefore, the intrinsic polarization of DH Cep is found to be less than $1 \%$ in all optical bands, but the trends of Fig. 1 will remain same.

The modulations in intrinsic polarization of DH Cep may be arising due to any of the following reasons:

1. asymmetric and extended circumstellar/binary envelopes

2. the presence of instabilities causing inhomogeneities in the massive-star winds (e.g. blobs)

3. the rotation of spots or non-radial stellar pulsations

4. modulations because of the binary motion

The variations in the polarization light curves should be strictly periodic due to reason (4) but the presence of the other three effects might also impose several intermittent periodicities (St.-Louis et al. 1987). Significant polarization variability seen in the B and V bands is probably due to the asymmetric circumstellar/binary envelopes.

Until now, the phase coverage of DH Cep is sparse. We are observing this source continuously during the current and future observation cycles to complete its polarization light curve. Our next aim is to estimate the parameters like inclination $i$, mass loss rate and the distribution of the scattering material around the binary stars by using available standard models (St.-Louis et al. 1987). The inclination of the binary systems can also be found from their photometric light curves. But this method requires a sufficiently high value of $i$ to yield eclipses, either involving the stars themselves or from the phase-dependent diminution of the light from one star as it orbits in the wind of the other star. On the other hand, the phase-dependent linear polarization modulations are used extensively for the low inclination $\mathrm{WR}+\mathrm{O}$ binaries. Once the value of $i$ is known, accurate masses of the binary components can be estimated, which is difficult otherwise. This method also provides reliable mass loss rates since intrinsic polarization is largely expected to be originating from the inner parts of the wind, where the density is large. Therefore, the uncertainties of the wind parameters in its outer parts, which are involved in other techniques of finding the mass loss rates like radio-based data, do not affect the results (St.-Louis et al. 1988). 

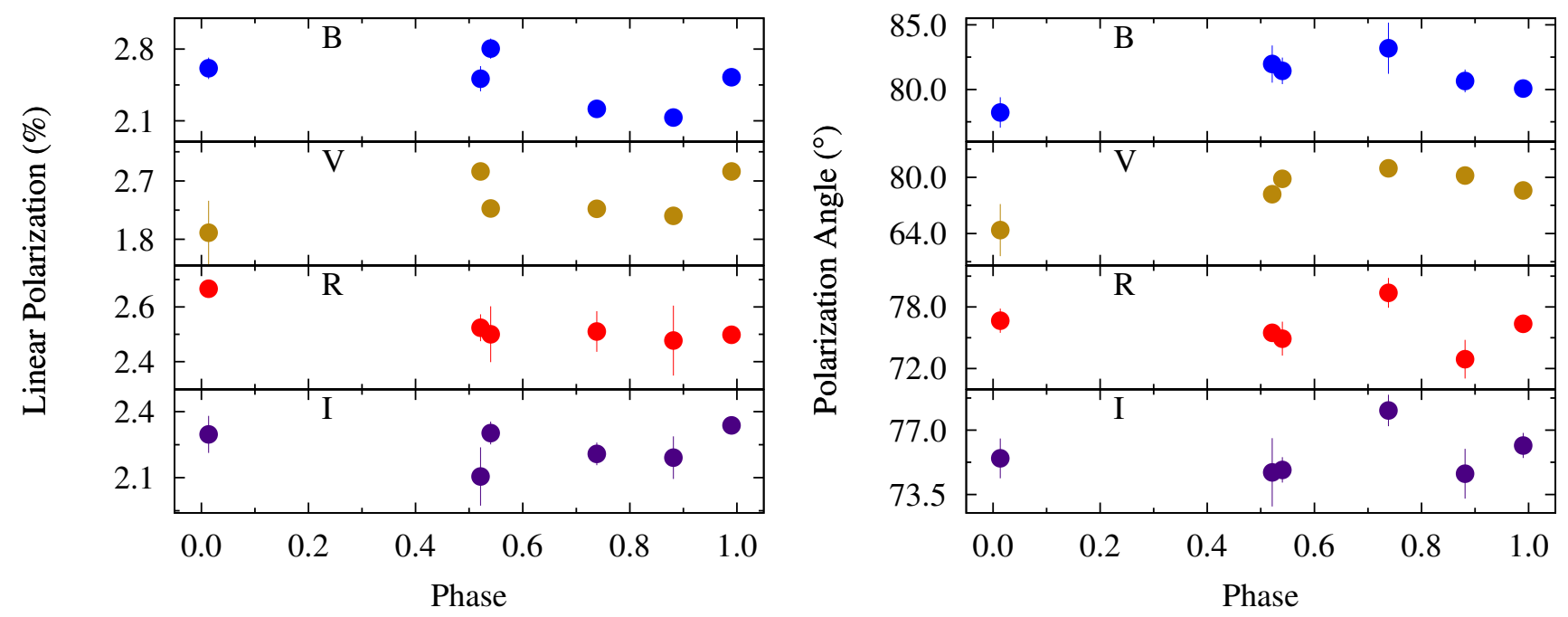

Figure 1: Linear polarization (left) and polarization angle (right) variation of DH Cep with the orbital phase in $\mathrm{B}, \mathrm{V}, \mathrm{R}$, and I filters running from top to bottom.

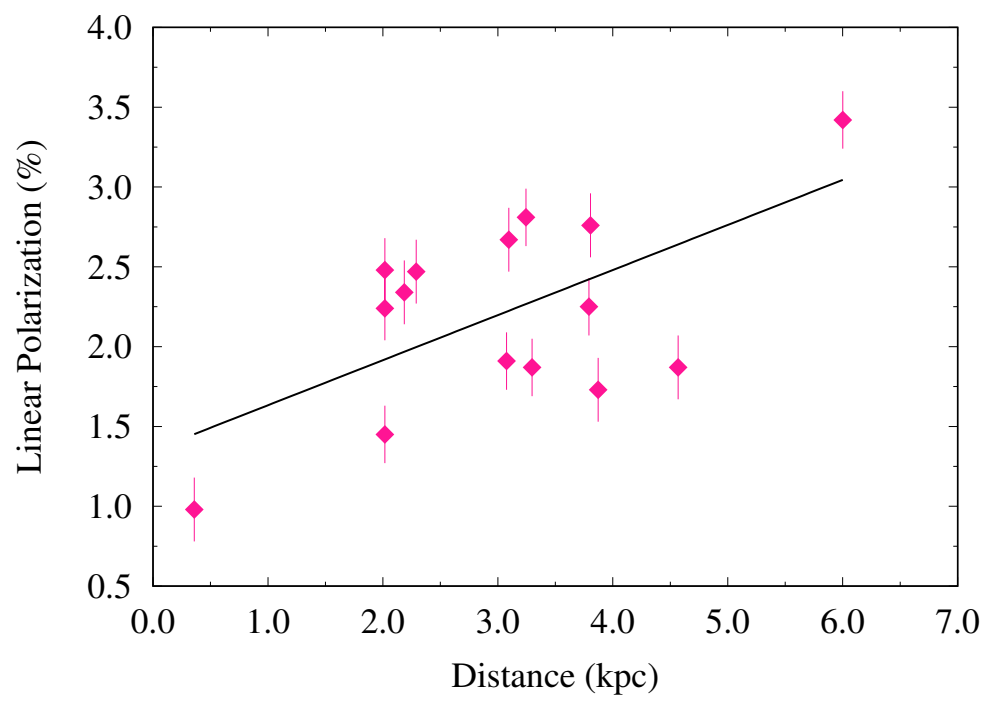

Figure 2: Linear polarization (V-band) as a function of distance towards the direction of DH Cep.

\section{References}

Bhatt H., Pandey J. C., Kumar B. et al. 2010, NewA, 15, 755

Drissen L., Moffat A. F. J., Bastien P. et al. 1986, ApJ, 306, 215

Heiles C. 2000, AJ, 119, 923

Lata S., Pandey A. K., Panwar N. et al. 2016, MNRAS, 456, 2505

Lindegren L., Hernández J., Bombrun A. et al. 2018, A\&A, 616, A2

Martins F., Mahy L., Hervé A. 2017, A\&A, 607, A82

Rautela B. S., Joshi G. C., Pandey J. C. 2004, BASI, 32, 159

Schmidt G. D., Elston R., Lupie O. L. 1992, AJ, 104, 1563

St.-Louis N., Drissen L., Moffat A. F. J. et al. 1987, ApJ, 322, 870

St.-Louis N., Moffat A. F. J., Drissen L. et al. 1988, ApJ, 330, 286

St.-Louis N., Moffat A. F. J., Lapointe L. et al. 1993, ApJ, 410, 342 